\title{
Editorial
}

\section{Nanostructured Materials for Microelectronic Applications}

\author{
Shoou-Jinn Chang, ${ }^{1}$ Teen-Hang Meen, ${ }^{2}$ Stephen D. Prior, ${ }^{3}$ \\ Artde Donald Kin-Tak Lam, ${ }^{4}$ and Liang-Wen $\mathrm{Ji}^{5}$ \\ ${ }^{1}$ Department of Electrical Engineering, Institute of Microelectronics, National Cheng Kung University, Tainan 701, Taiwan \\ ${ }^{2}$ Department of Electronic Engineering, National Formosa University, Yunlin 632, Taiwan \\ ${ }^{3}$ Aeronautics, Astronautics and Computational Engineering, University of Southampton, Southampton SO17 1BJ, UK \\ ${ }^{4}$ College of Physics and Information Engineering, Fuzhou University, Fuzhou 350116, Fujian, China \\ ${ }^{5}$ Institute of Electro-Optical and Materials Science, National Formosa University, Yunlin 632, Taiwan
}

Correspondence should be addressed to Shoou-Jinn Chang; changsj@mail.ncku.edu.tw

Received 29 January 2014; Accepted 29 January 2014; Published 6 March 2014

Copyright (c) 2014 Shoou-Jinn Chang et al. This is an open access article distributed under the Creative Commons Attribution License, which permits unrestricted use, distribution, and reproduction in any medium, provided the original work is properly cited.

The Scope of the Special Issue. Nanostructured materials are a new class of materials, having dimensions in the $1 \sim 100 \mathrm{~nm}$ range, which provide one of the greatest potentials for improving performance and extended capabilities of products in a number of industrial sectors. Nanostructures can be divided into zero-dimensional (0D when they are quantum dot or nanoparticle structures), one-dimensional (1D when they are elongated), and two-dimensional (2D when they are planar) based on their shapes. The recent emphasis in the nanomaterials research has been on $1 \mathrm{D}$ nanostructures perhaps due to the intriguing possibility of using them in the majority of short-term future applications. The most successful examples are seen in the microelectronics, where "smaller" has always meant a greater performance ever since the invention of transistors, for example, higher density of integration, faster response, lower cost, and less power consumption. In recent years, applications of nanostructured nanomaterials on microelectronic devices have been a highly developing field, due to the flexibility and the light weight for daily use, which has the potential to be deployable. Therefore the fields of nanostructure devices have been the subject of reviews.

With the continuous downscaling of material technologies, reliability is more and more becoming a major bottleneck. First of all the electric fields and mechanical stress have increased continuously and are now reaching the maximum values that can be allowed for reliable operation. At this time, we are introducing nanomaterials and a new design to maintain the effective performance scaling. Some materials like the low carbon alloy steel, transparency conducting AZO films, piezoelectric material, AlN flake, and titanium dioxide films have already been introduced and under investigation. These materials and devices often have unknown reliability behavior and/or introduce new failure mechanisms. In addition, the market is continuously demanding higher reliability levels for present technologies. The chance to share and discuss these crucial nanostructured materials for microelectronic developments is very important.

This special issue selects 9 papers about materials for microelectronic applications and other related fields. This special issue enables interdisciplinary collaboration between material science and engineering technologists in the academic and industrial fields.

Shoou-Jinn Chang Teen-Hang Meen Stephen D. Prior Artde Donald Kin-Tak Lam Liang-Wen Ji 

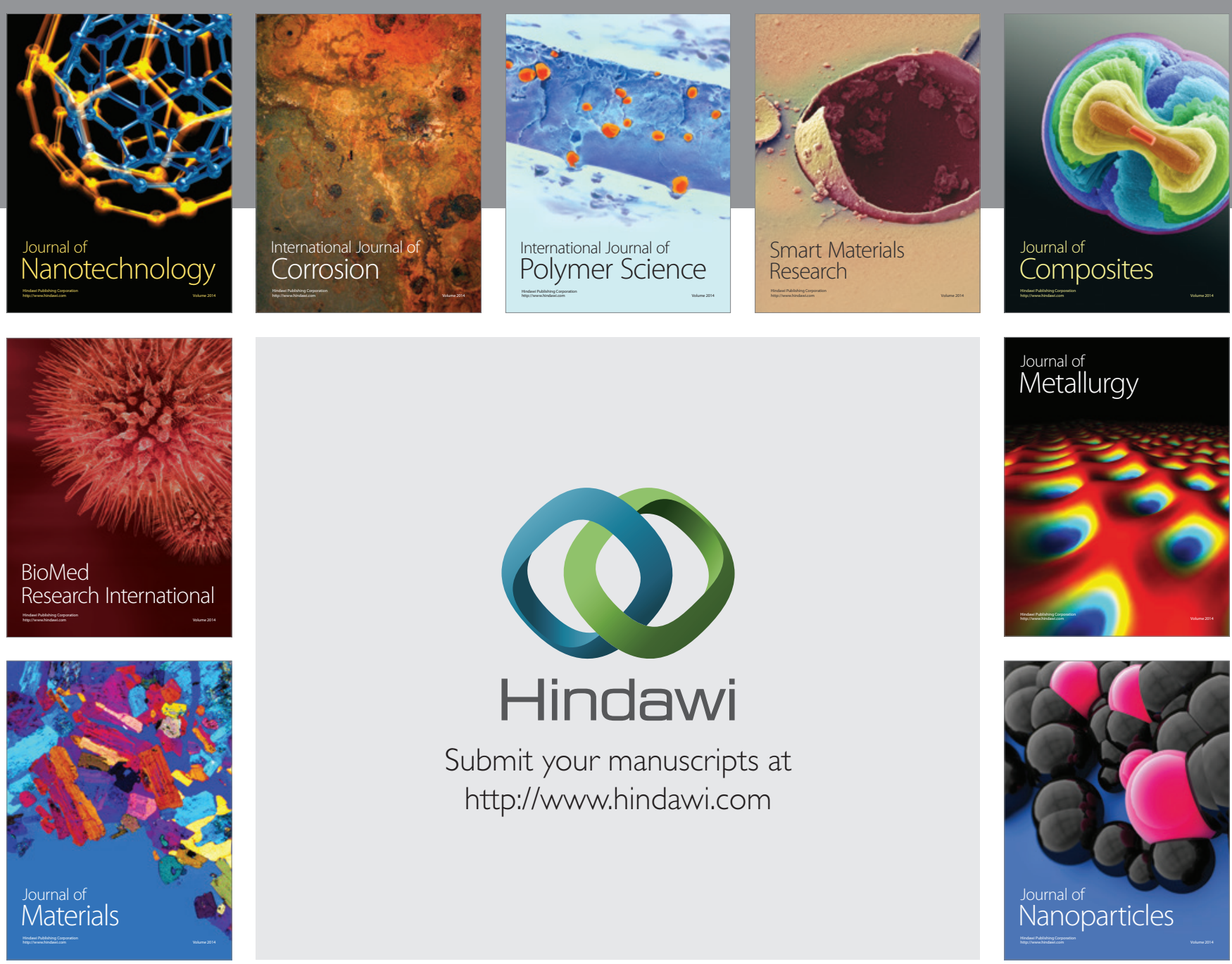

Submit your manuscripts at http://www.hindawi.com
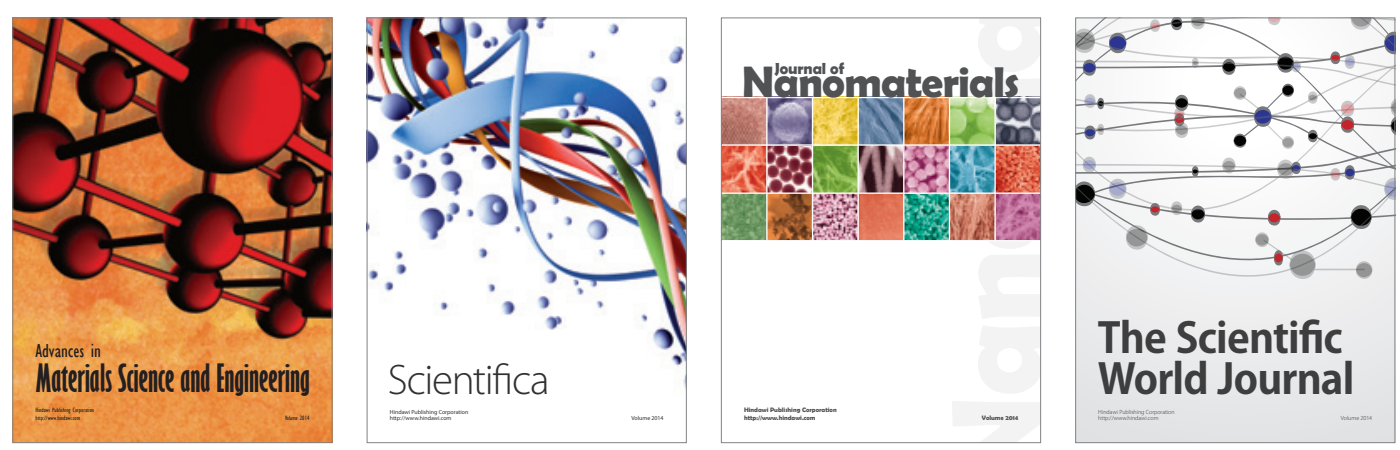

\section{The Scientific World Journal}
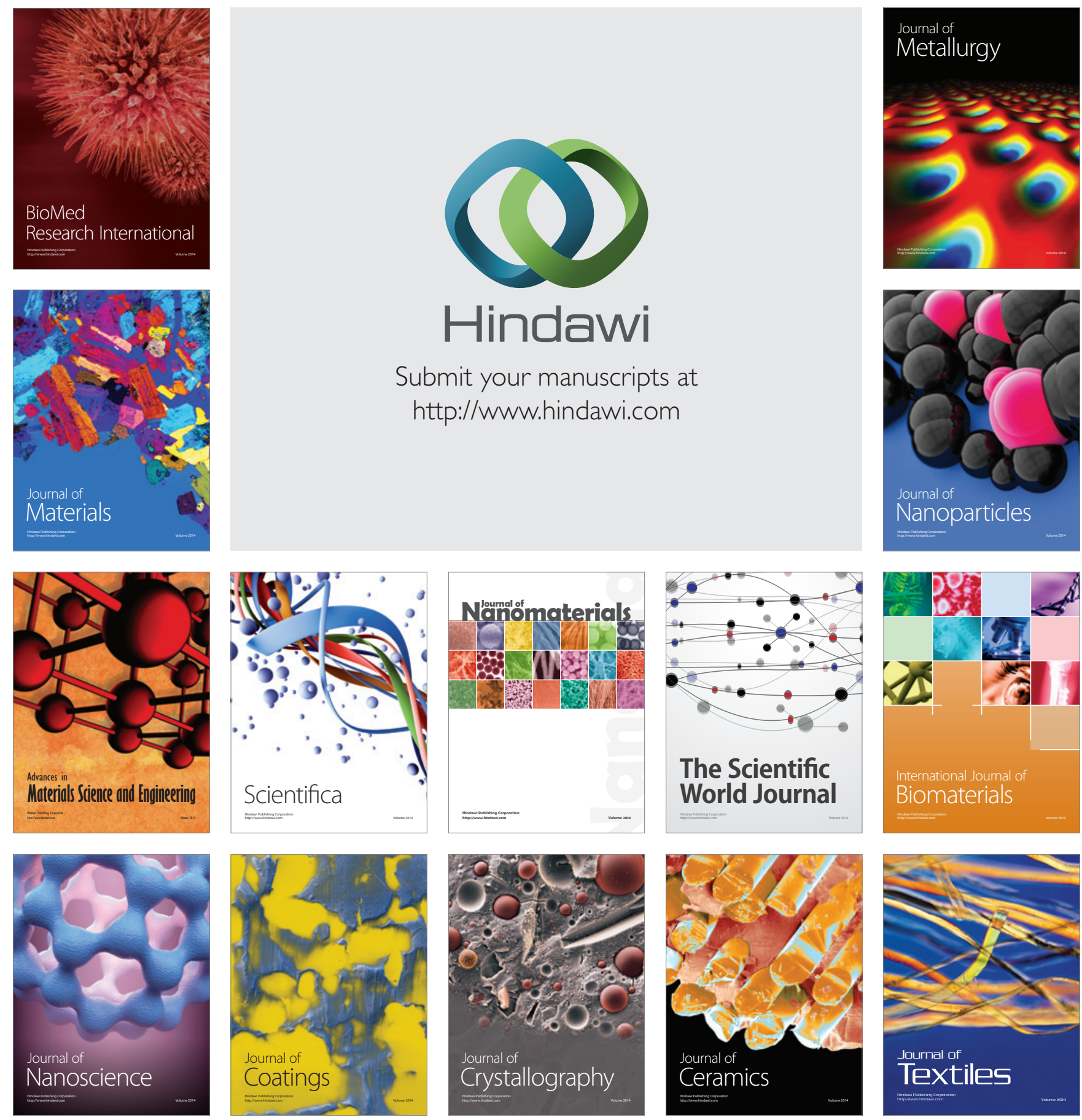